\title{
MEASURES ON NON-SEPARABLE METRIC SPACES
}

\author{
BY \\ R. M. DUdLEY ${ }^{1}$

\section{Introduction}

The main purpose of this note is to give a simpler and more general definition of "weak" or "weak-star" convergence of certain measures on nonseparable metric spaces, and to prove its equivalence with the convergence introduced in [1] for the cases considered there.

Let $(S, d)$ be a metric space. Let $\beta$ or $\Theta(S)$ be the class of all Borel sets in $S$, i.e. the smallest $\sigma$-algebra containing all the open sets. One can safely assume that a finite, countably additive measure on $B$ is concentrated in a separable subset [2]. It has seemed useful to consider finite, countably additive measures on metric spaces, not concentrated in separable subsets, defined on some, but not all, Borel sets [1]. Specifically, one can use the $\sigma$-algebra $\mathfrak{u}$ or $\mathcal{u}(S)$ generated by the open balls

$$
B(x, \varepsilon)=\{y \in S: d(x, y)<\varepsilon\}
$$

for arbitrary $x$ in $S$ and $\varepsilon>0$. Examples of finite measures on $\mathcal{u}$ not concentrated in separable subsets are the probability distributions of distribution functions of "empirical measures" [1]. For a simpler example, let $S$ be uncountable and $d(x, y)=1$ for $x \neq y$. Then $\mathcal{u}$ consists of countable sets, which we give measure 0 , and sets with countable complement, which we give measure 1.

If $S$ is separable, then all open sets are in $u$ by the Lindelöf theorem, hence $\mathfrak{u}=\circledast$. I don't know whether $\mathfrak{u}$ is always strictly included in $\leftrightarrow$ for $S$ nonseparable, but it is in the cases mentioned above, and under the following conditions:

Proposition. Suppose that the smallest cardinal of a dense set in $S$ is $c$ (cardinal of the continuum). Then $\mathcal{u}$ has cardinal $c$ and $\Theta$ has cardinal $2^{c}$. Hence $\mathfrak{u}$ is strictly included in $B$.

Proof. Let $A$ be a dense set in $S$ of cardinal $c$. Let $G$ be the class of balls $B(x, r)$ with $x$ in $A$ and $r$ (positive) rational. We show that $G$ generates $\mathcal{U}$. Let $x \in S, r>0$. Let $x_{n} \in A, x_{n} \rightarrow x$. We can assume $d\left(x_{n}, x\right)<r$ for all $n$. Let $r_{n}$ be positive rational numbers such that $r_{n} \rightarrow r$ and $r_{n}<r-d\left(x_{n}, x\right)$ for all $n$. Then

$$
B(x, r)=\bigcup_{n=1}^{\infty} B\left(x_{n}, r_{n}\right),
$$

showing that $G$ generates $\mathcal{~}$.

Received July 1, 1966.

1 This research was supported in part by a National Science Foundation grant, and was presented to the International Congress of Mathematicians in Moscow, August 1966. 
Let $\omega$ be the cardinal of the set of all integers. Then $G$ has cardinal at most $\omega c$, and $\omega c=c$. Hence the class of complements of sets in $G$ has cardinal at most $c$. The class of countable unions of elements of $G$ has cardinal at most equal to $c^{\omega}$, and

$$
c^{\omega}=\left(2^{\omega}\right)^{\omega}=2^{\omega^{2}}=2^{\omega}=c .
$$

Using transfinite induction, we obtain that the cardinal of $\mathcal{u}$ is at most $\boldsymbol{\aleph}_{1} c$ (where $\boldsymbol{\aleph}_{1}$ is the least uncountable cardinal; we are assuming the axiom of choice, but not the continuum hypothesis). Now $\boldsymbol{N}_{1} c=c$. Since each onepoint set in $S$ clearly belongs to $\mathcal{u}$, the cardinal of $\mathcal{u}$ is exactly $c$. The cardinal of $B$ is exactly $2^{c}\left[3\right.$, Remark 3.7 p. 106] and $c<2^{c}$. Thus $\mathcal{u}$ is properly included in $B$, q.e.d.

If in the statement of the above proposition we replace $c$ by another uncountable cardinal $\alpha$, then the proof goes through except that possibly $\alpha<\alpha^{\omega}$, which will happen e.g. if $\alpha=\aleph_{\omega}\left[3\right.$, p. 100], but not if $\alpha=2^{\beta}$ for some (infinite) $\beta$. When $\mathcal{u}$ and $\leftrightarrow$ have the same cardinal, it remains unclear whether they are equal.

It should be noted that the $\sigma$-algebras $\mathcal{u}$ in non-separable metric spaces have certain unpleasant properties. For example, they are not always preserved by homeomorphisms or even by uniform isomorphisms. Also, they are not always preserved by "relativization" to a subset of $S$ with the same metric. Finally, if one takes a cartesian product of two metric spaces $S$ and $T$, with any of the usual metrics for the product topology, $\mathcal{U}(S \times T)$ may not even contain all "rectangles" $A \times B$ where $A \in \mathcal{U}(S), B \in \mathcal{U}(T)$.

The Borel $\sigma$-algebras are superior in all these respects, although $B(S \times T)$ may not be generated by the rectangles whose sides are Borel sets. Of course, the Borel $\sigma$-algebras are generally too large to carry a finite measure with non-separable support. One might hope for a $\sigma$-algebra which, like $u$, would allow such measures, but which had better "functorial" properties.

\section{Measures on $\mathfrak{u}$}

Let $M(S, \mathcal{u})$ be the set of all finite, countably additive, real-valued set functions (signed measures) on $\mathfrak{u}, M^{+}(S, \mathfrak{u})$ the set of elements of $M(S, \mathfrak{u})$ with nonnegative values, and $P(S, \mathcal{u})$ the set of elements of $M^{+}(S, \mathfrak{u})$ with total mass 1 (probability measures).

In [1], "weak-star" convergence of a sequence in $M^{+}(S, \mathcal{u})$ to a Borel measure $\mu$ was defined as convergence of the upper and lower integrals of every bounded continuous function $f$ to $\int f d \mu$. Here we define a natural convergence in $M(S, \mathcal{u})$ and prove that if $S$ is complete, the new convergence agrees with the old one whenever the latter is defined (if $\mu$ has separable support, which, as noted above, practically follows from $\mu$ being a Borel measure).

Let $\mathfrak{C}(S)$ be the Banach space of all bounded, continuous, real-valued functions on $S$ with supremum norm \|\|$_{\infty}$. Let $C(S, \mathcal{u})$ be the closed linear 
subspace of $\mathcal{u}$-measurable elements of $\mathfrak{e}(S)$. Then any $\mu$ in $M(S, \mathcal{u})$ defines a bounded linear functional

$$
f \rightarrow \int f d \mu
$$

on $\mathcal{e}(S, \mathfrak{u})$. Then on $M(S, \mathfrak{u})$, we have the "weak-star" topology of pointwise convergence on $\mathfrak{e}(S, \mathfrak{U})$. (Note that $M(S, \mathfrak{u})$ is a proper subset of the dual space $\mathfrak{e}(S, \mathfrak{u})^{*}$ unless $S$ is compact.)

Given a real-valued function $f$ and a measure $\mu$ we define the usual upper and lower integrals:

$$
\begin{gathered}
\int^{*} f d \mu=\inf \left\{\int h d \mu: h \geq f, \int h d \mu \text { defined }\right\}, \\
\int_{*} f d \mu=\sup \left\{\int g d \mu: g \leq f, \int g d \mu \text { defined }\right\} .
\end{gathered}
$$

Theorem. Suppose $(S, d)$ is a complete metric space, $\left\{\mu_{n}\right\}$ is a sequence of elements of $M^{+}(S, \mathcal{U})$ and $\mu$ in $M^{+}(S, \mathcal{U})$ is concentrated in a separable subspace. Then $\mu_{n} \rightarrow \mu$ for the weak-star topology on $M(S, \mathcal{U})$ if and only if

$$
\lim _{n \rightarrow \infty} \int^{*} f d \mu_{n}=\lim _{n \rightarrow \infty} \int_{*} f d \mu_{n}=\int f d \mu
$$

for every $f$ in $\mathcal{C}(S)$.

Proof. "If" holds since the upper and lower integrals of functions in $\mathcal{e}(S, \mathcal{U})$ are integrals.

To prove "only if", suppose $\mu_{n} \rightarrow \mu$ on $\mathcal{C}(S, \mathcal{u})$ and $f$ is in $\mathcal{C}(S)$. Since $\mu$ has separable support it has a natural extension to all Borel sets. We may assume $\|f\|_{\infty} \leq 1$ and $\mu_{n}(S) \leq 1$ for all $n$. Let $\varepsilon$ be given, $0<\varepsilon<1$. By Ulam's theorem [4], there is a compact set $K$ such that $\mu(S \sim K)<\varepsilon$. Choose $\delta>0$ so that $d(x, y)<\delta$ and $x$ in $K$ imply $|f(x)-f(y)|<\varepsilon$. Let $C$ be countable and dense in $K$. Let

$$
d(y, K)=\inf _{x \in K} d(x, y)=\inf _{x \in C} d(x, y) .
$$

Then $d(\cdot, K)$ is $\mathcal{U}$-measurable and continuous (in fact,

for all $y$ and $z$ ). Let

$$
|d(y, K)-d(z, K)| \leq d(y, z)
$$

Then $g \in \mathfrak{e}(S, \mathcal{u})$, so

$$
g(y)=\min (1,4 d(y, K) / \delta) .
$$

$$
\int g d \mu_{n} \rightarrow \int g d \mu<\varepsilon
$$

Let $F$ be a finite subset of $K$ such that for any $x$ in $K, d(x, z)<\delta / 4$ for some $z$ in $F$. Let

$$
\phi(t)=\varepsilon t / \delta, \quad 0 \leq t \leq \delta / 2
$$




$$
=2, \quad t \geq \delta
$$

and let $\phi$ also be linear in the interval $[\delta / 2, \delta]$. Let

$$
\begin{aligned}
& u(x)=\min (1, \min (f(z)+\varepsilon+\phi(d(x, z)): z \epsilon F)), \\
& v(x)=\max (-1, \max (f(z)-\varepsilon-\phi(d(x, z)): z \epsilon F)) .
\end{aligned}
$$

Then clearly $u, v \in \mathfrak{e}(S, \mathfrak{u})$. Let

$$
W=\{x: d(x, w)<\delta / 4 \text { for some } w \text { in } K\} .
$$

For any $x$ in $W, d(x, z)<\delta / 2$ for some $z$ in $F$, so

Thus

$$
|f(x)-f(z)|<\varepsilon \text { and } \phi(d(x, z))<\varepsilon .
$$

$$
u(x) \leq f(z)+2 \varepsilon \leq f(x)+3 \varepsilon .
$$

Given $x$, let $G_{x}$ be the set of all $z$ in $F$ such that $d(x, z)<\delta$. Then $f(x) \leq$ $f(z)+\varepsilon$ for all $z$ in $G_{x}$, while for $z$ in $F \sim G_{x}, \phi(d(x, z))=2$. Thus $f(x) \leq u(x)$ for all $x$ in $W$. Likewise

$$
f(x) \geq v(x) \geq f(x)-3 \varepsilon
$$

for all $x$ in $W$. Now since $W \in \mathfrak{U}$,

$$
\begin{aligned}
\int^{*} f d \mu_{n} & \leq \int_{W} u d \mu_{n}+\mu_{n}(S \sim W), \\
\int_{*} f d \mu_{n} & \geq \int_{W} v d \mu_{n}-\mu_{n}(S \sim W), \\
\lim \sup \int^{*} f d \mu_{n} & \leq \lim \sup \int_{W} u d \mu_{n}+\varepsilon, \\
\lim \inf \int_{*} f d \mu_{n} & \geq \lim \inf \int_{W} v d \mu_{n}-\varepsilon,
\end{aligned}
$$

and

$$
\lim \sup \int_{W}(u-v) d \mu_{n} \leq 6 \varepsilon,
$$

so

$$
\lim \sup \int^{*} f d \mu_{n}-\lim \inf \int_{*} f d \mu_{n} \leq 8 \varepsilon .
$$

Since an upper integral is greater than a lower integral of the same function, the limits of $\int^{*} f d \mu_{n}$ and $\int_{*} f d \mu_{n}$ exist and are equal. These limits are also approached by $\int_{W} f d \mu$ as $\varepsilon \rightarrow 0$ (of course, $W$ depends on $\varepsilon$ ), thus they equal $\int f d \mu$, q.e.d.

\section{REFERENCES}

1. R. M. DudLex, Weak convergence of probabilities on nonseparable metric spaces and empirical measures on Euclidean spaces, Illinois J. Math., vol. 10 (1966), pp. 109-126. 
2. E. Marczewski and P. Sikorski, Measures in nonseparable metric spaces, Colloq. Math., vol. 1 (1948), pp. 133-139.

3. A. H. Stone, Cardinals of closed sets, Mathematika, vol. 6 (1959), pp. 99-107.

4. S. ULAM AND J. C. OxтовY, On the existence of a measure invariant under a transformation, Ann. of Math., vol. 40 (1939), pp. 560-566.

UNIVERSity of CALIForNia

Berkeley, California

Massachusetts Institute of Technology

Cambridge, Massachusetts 